\title{
Fourth Day of Creation: The Proto-history of Young Stars, Star Streams, and Exoplanets Near the Sun
}

\author{
Virginia Trimble ${ }^{1}$ \\ ${ }^{1}$ Department of Physics and Astronomy, University of California, Irvine, CA, USA
}

\begin{abstract}
Items of scientific knowledge at any moment in time have pre-histories when they were debated, doubted, or absolutely denied. The examples considered here are the admitted facts that star formation is an on-going process in the Milky Way, that there are young moving groups (the products of young star clusters in the process of dissolution and perhaps more complex processes), and that planets orbiting other stars are common. It is hard to imagine any of these ceasing to be part of core astronomical knowledge, but you are advised not to place large bets on this.
\end{abstract}

Keywords. Star Formation, Moving Groups, Star-Streams, Exoplanets

\section{Introduction}

The after-dinner version of this approach to the topic of the conference included a large number of images of ancient cosmologies and people, early published texts, and handwritten letters. Not one of them is here, because I do not own copy-rights to the first two classes, and the latter two classes are almost unreadable. However, some attempt is made to convey the flavor of the presentation, with references to where much of the information can be found.

\section{Young Stars and On-Going Star Formation}

Vaya'ash Elohim et shney hamorot hag'dolim...And G.d made two great lights, the greater light to rule the day and the lesser light to rule the night. V'et hakochabim. And also the stars... Vayechi erev, vayechi boker, yom rivi'i. And there was evening and there was morning. A fourth day. This version makes clear that the stars are somewhat incidental to the solar system and are all the same age. The writers of Genesis got the time scale wrong by a factor of a million or so, but the idea that everything is the same age lasted long.

In contrast, for the ancient Egyptians, the Chinese, and the Greeks, star formation continued into their present. The shafts extending from the king's burial chamber in Cheops' pyramid were intended for his ka to rise and join the stars; you have undoubtedly heard of the Chinese recording what we now call supernovae as "guest stars;" and the Greeks even provide a tentative ordering of what formed first. By this I mean Greek mythology, not the much less picturesque equants and deferents of Ptolemy and his followers. Careful reading of these leads us to the conclusion that the gods first put the Hyades (the daughters of Atta) in the sky and then the Pleiades, apparently to save them from being chased by Orion, who, however, followed them into the sky. He was joined later by his dogs Canis Major and Canis Minor. This does not correspond to our modern 
ordering, though the Pleiades are younger than the Hyades, and the Orion Nebula stars younger than either.

Through the 18th, 19th, and into the 20th century there were at least half a dozen swings back and forth between these two views of "all at once, long ago" and "ongoing process" (Trimble 1997). The last adherents of "all at once" were probably de Vaucouleurs, who wanted to form galaxies and stars in a coasting phase of a universe with a cosmological constant as late as 1958, and David Layzer with his 1964 cold big bang. Herschel over his lifetime rode both horses in succession, eventually settling on spiral nebulae as systems in formation. Russell, Dugan, and Stewart had "ongoing" in the first, 1927 edition of their two volume classic text and "all at once" in the 1938 second edition. Swings to "all at once" came from the first understanding of thermodynamics (if everything is running down it must all have started in the past) and later from estimates of stellar lifespans from the recognition of hydrogen fusion as the primary energy source. The mistake there was the feeling that, if most things are the same age (stars like the sun; the Hubble time; the oldest earth rocks and meteorites), then probably everything is the same age.

Swings toward "on-going" had come from Herschel's nebulae and Russell's giant and dwarf theory of stellar evolution in 1913. You might have expected Huggins's discovery of gas clouds, an obvious source of material for new stars, to have settled the issue in the 1860 's, but he said not. Huggins's own view was that the proto-stellar material advocated by Herschel would have to contain all the elements found in the sun, while he recognized only hydrogen, nebulium, and something he thought was nitrogen, but was not, in the gaseous nebulae.

Thus the issue was settled observationally when V.A. Ambartsumyan and Adriaan Blaauw pointed to expanding groups of stars that included OB stars and associated gas. The discoveries of $\mathrm{T}$ Tauri stars and Herbig-Haro objects aided the process. In 1948, Lyman Spitzer was still putting much star formation in the early universe (with incomprehensible and perhaps different physics), but said that the supergiants must be young since hydrogen fusion could not have kept them that bright for long. Cecilia Payne Gaposchkin, writing for general readers in 1952, said, "We can even toy with the idea that stars are being born continuously; and the places where the young stars are found suggest very definitely that they are born of the interstellar dust."

Because papers in 1939 by Spitzer and by Fred Hoyle had come so close to suggesting on-going star formation as the dominant process, I wrote to them in 1996 asking for their reflections on that time and subject. Both answered. Hoyle reminded me that, even now, the origin of spectroscopic binaries from clouds is difficult to understand and also that, in 1939, the astronomical world did not believe in an interstellar medium containing hydrogen. What we now call Bondi-Hoyle accretion (though Lyttleton was co-author on one of the papers) was intended to bulk up old, low mass stars into OB's and supergiants.

Spitzer's response told of a talk he had given at Harvard in 1939 and a paper submitted to ApJ later that year incorporating "certain dynamical processes in the interstellar medium" that would have led to star formation. These were objected to as "too speculative," and the paper was published without that material. He also answered my next letter, in which I had, of course, asked who the objectors were. Spitzer declined to answer "since one of these is still very much alive." But he signed as Lyman, to show we were still friends. I was never able to think of anyone this could have been except Jesse Greenstein, but at lunch the next spring Jesse said no. Only some massive unsealing of ApJ records from 1939 (which may not even still exist) could now tell us. Spitzer has himself told the non-secret parts of the story in his chapter in volume 7 of the Kuiper Compendium, Stars and Stellar Systems. 
The heroes whose pictures appeared at this point were, of course, Ambartsumyan, Blaauw, and Spitzer.

\section{Moving Groups and Star Streams}

Blaauw's (1952) study of the stars of the Zeta Persei group and of surrounding O to B5 stars provides a natural transition to this second topic. His figure shows proper motions after the subtraction of the mean of the group, thus revealing the expansion and a dynamical time scale of only about 10 million years, so there are both moving groups and ongoing star formation. But we can look much farther back.

Relative proper motions of stars were discovered by Halley, comparing his positions with those of Ptolemy. By 1783 enough numbers had accumulated for William Herschel to declare that there was a component from the reflex of solar motion, meaning that we are moving more or less toward Hercules, a result which stands today (North 2008 pp. 423, 436). But it was Richard Proctor $(1869,1870)$ who concluded that published catalogues were concealing another effect. Stars were generally listed in declination zones, but, he said, take a star map and put little arrows to represent proper motions and you will find what he called star drift.

Proctor drew attention to the drift in Taurus (the Pleiades and its neighbors) toward the north-west, in Gemini and Cancer toward the south-east, and Leo toward Cancer. These are still more or less, he said, in the direction of solar motion (and there have been frequent claims for a large solar association). Five of the seven bright stars in Ursa Major and three in Arietes drift in the opposite direction. He concluded that it was at least possible that the local stellar system was in rotation around the rich cluster $\mathrm{h}$ and $\chi$ Persei, and he looked forward to the "stellar proper motions of recess or approach" to be expected from Mr. Huggins, now called radial velocities. For me, the symbol will always be $V_{r}$, but most speakers at IAU Symposium 314 used RV (well, recreational vehicles also display proper motions).

Angular proper motions and indeed radial velocities accumulated and by 1927 Russell, Dugan, and Stewart recognized moving clusters Ursa Majoris, the Hyades (with distance determination), Sco-Cen, Perseus, Pleiades, Coma Berenices, and Praesepe in Cancer (some of these in common with Proctor's systems).

Meanwhile, however, there had arisen a confusorium, with Kapteyn in 1905 describing the large system of peculiar motions as two interlaced streams moving in opposite directions. Karl Schwarzschild soon after put forward his velocity ellipsoid. The two pictures are essentially equivalent said RDS, though the latter retains some currency as a way of characterizing the asymmetries of stellar motions perpendicular to the galactic disk toward and away from the galactic center, and in the direction of galactic rotation. What Kapteyn and Schwarzschild were seeing, through a telescope darkly, was galactic rotation of the disk, but not the halo traced by globular clusters and other old, high-proper motion stars. Schwarzschild and Hertzsprung (1913) pointing out that Preasepe moves with the Hyades and Wilson's (1932) discovery that $2 \%$ of stars near the sun share the motion of the Hyades and constitute the Taurus group to be found all over the sky, are also part of this story. Plots of star streaming from RDS that I described as "fairly incomprehensible" gave their caption if not their plot to a couple of diagrams shown on Friday by others.

Analyses of stellar peculiar motions were often described as measuring the solar motion relative to some specific population right down to the time when Hubble used the population of external galaxies in 1929 and found something else. 
But we leave it to Oort $(1926,1932)$ to sort out the large scale Milky Way patterns so as to be able to hurry on to Olin Jeuck Eggen. His life (see Eggen 1993) counts as nearly indescribable. The picture of him I showed was his own choice for that memoir (the other three pictured heroes were Kapteyn, K. Schwarzschild, and Oort), though Mike Bessell has one he likes much better. Bessell also updated the "two carburetors" story (Trimble et al. 2001) with the topper that, when other astronomers acquired cars with two, Eggen claimed three.

Starting in 1957, Eggen indicated that the Taurus Group included 1\% of FK5 stars and was part of a group moving through the solar neighborhood at $44 \mathrm{~km} / \mathrm{sec}$. Later studies found superclusters associated with Sirius, Hyades, Pleiades, IC 2391, and NGC 2516 and 2287 (both part of the Pleiades group). Intermediate summaries are Eggen (1965, 1993) up to the final Eggen (1998) in which he concluded that physically meaningful groups might share only motion, not the ages of the stars, compositions, or directions in the sky. Should much of this be classified with his "trend lines" in HR diagrams from earlier work? I have certainly thought so in the past (Trimble (2000). But, in 2004, when SDSS data began to recognize disrupted dwarf satellites as star streams in the Milky Way (Navarro et al. 2004) my instantaneous reaction was "Egad! Eggen was right!" The possibility deserves a closer look by some young, kindly person!

Looking ahead, there will, of course, be more star streams coming out of surveys from SDSS and GAIA. GAIA also finds Exoplanets, which brings us to the next section. The fifth person in the photograph I showed was Jim Gunn (in 1971), the founding spirit of SDSS.

\section{Exoplanets, or, All My Errors are Systematic}

A systematic error is the scientific equivalent of doing the same thing over and over again and expecting a different result (one of psychology's definitions of insanity). This is no longer a problem for Exoplanets. The right path was foreshadowed in Zuckerman and Song (2004) whose abstract ends by saying, "These young groups (ages 8-50 Myr) along with other nearby, young stars will enable imaging and spectroscopic studies of the origin and early evolution of planetary systems," and there is no doubt that IAUS 314 marks a major step forward on that trajectory. But let's look back, in order, at direct imaging, radio and optical radial velocities, transits, and finally astrometry, the most interesting, heroic, and tragic of the tales.

\subsection{Direct Imaging}

William Herschel is said several places to have suspected a third light in 70 Oph, when the stars were about $4.5^{\prime \prime}$ apart and each of naked eye magnitude. By the last day of IAUS 314 there were "about 10" directly-imaged Exoplanets, not all from HST, but nearly all involving great cleverness in adaptive optics, coronagraphy, and other ways of cheating mother nature. A few retractions have not, I think, cost anybody life, limb or reputation.

\subsection{Radio Radial Velocities}

The trick is to time pulsars very, very accurately. Bailes et al. (1991) in this way thought briefly that they had found evidence for a six-month-period oscillation in the radial velocity of PSR 1829-10. Well, yes, but it was the earth moving in and out of the sun's gravitational field They had allowed for special relativistic (Doppler) but not general relativistic (gravitational redshift) effects.

Triumph was, however, waiting just around the calendric corner for Wolszczan and Frail (1992) who found first one, then two (and now I think there are three) planets 
orbiting PSR 1957+12. Masses and orbits are both small compared either to Jupiter or later "hot Jupiters," and they are probably bits of broken-off neutron star material, now mostly iron, rather than habitable rocks and volatiles. Pulsar timing carries on at several observatories, but has not ever quite struck the same kind of iron again.

\subsection{Optical Radial Velocities (winners old and new)}

Black (1995), who called his review "Completing the Copernican Revolution," had the misfortune to write just before the Geneva and Lick groups announced 51 Peg b. Published studies at the time included Campbell et al. (1988) who had found a 1-5 Jupitermass planet orbiting $\epsilon$ Eridani (an early Drake candidate for SETI searches), now I think generally not counted, and Latham et al. (1989) who had certainly found something, but not a planet or even a brown dwarf, unless the system is much more nearly edge-on than other data suggested.

The winners (Mayor and Queloz 1995 and Marcy and Butler 1996) won big, with multiple prizes to their names and, within a decade, enough planets for statistical analysis, a large fraction found by their methods and groups (Jones 2004). I had the good fortune to address "The Quest for Other Worlds" at that same conference (Trimble 2004), providing a list of 23 possible detection methods. Of those I then thought dubious, gaps in YSO accretion disks now count as quite reliable, and, according to a comment by Ben Zuckerman, pollution of host star atmospheres has definitely been recognized among white dwarfs (Zuckerman 2015).

\subsection{Transits}

At peak, there were more than 20 groups looking for transits by Exoplanets (dimming of a star when the planet crosses its face, Charbonneau et al. 2004). Many had come up empty, simultaneously revealing a puzzling brown dwarf desert around normal stars. And the "detections" included a frightening cohort of false positives, 136 of 137 in one OGLE search (Torres e al. 2004). Eclipsing binaries, with grazing incidence or blended on the sky with a bright, non-varying star were the main sources. Meanwhile, the answer was lurking in the literature in a journal much less cited than Nature or ApJ Letters (Borucki and Summers 1984). "Go up, young man," was the answer, and though Kepler has also had its false positives, spot checking by radial velocity methods shows they are very few among the 3000 candidates to date (Borucki 2015). None of the discoveries is quite an earth-mass planet in the liquid-water zone of a sun-like star, but some are not far off, and Kepler has also been the source of most of the multi-planet systems. The record to date is probably 7 , organized in a size-period combination nothing like our solar system.

\subsection{Astrometry}

Relying on the same physics as radial velocity searches but in the plane of the sky instead of perpendicular to it, this is the oldest method, connected with the saddest stories and very few successes. The method works and led to the discoveries of Sirius B and Procyon B, both later imaged. That it might be extended into the planetary range was the thought of Capt. William Steven Jacobs (Jacobs 1855), who suspected he might have seen a small wiggle in the binary orbit of $70 \mathrm{Oph}$. Jacob is not obscure, in the sense of being represented in the (British) Dictionary of National Biography. He received however, little credit for his idea, though he was director at Madras (now Chennai) for many years, where Pogson (of the magnitude scale and also unfairly obscure) was a staff member and later Jacobs's successor. The saga of his last return to India, via 100 days under sail and leading to his death a few weeks later in Poonah, is found in a diary kept by his daughters. The hunt and peck method of using Google (etc) will find this via a 
Jacobs family genealogy. The meals described for the voyage leave one feeling that scurvy or some similar deficiency must have contributed to his death.

Next comes Thomas Jefferson Jackson See, who also looked at 70 Oph and thought (or anyhow claimed) that he had seen astrometric evidence for a planet around the secondary (See 1896). Chamberlin (of the Chamberlin-Moulton hypothesis) showed the triad would be unstable, and so obstreperous did See become (see See 1899, sorry!) that he was forbidden ever again to submit a paper there. He lived to a ripe old, perhaps even happy old, age, mostly on Mare Island, and received a rather nice obituary in Sky 8 Telescope (Ashbrook 1962) as well as in the New York Times and Physics Today. He belonged, at peak, to at least 25 professional scientific societies in at least 5 countries.

Later players in the astrometric tournament are less colorful, but generally not less unsuccessful. Wrong orbits were reported for 70 Oph by Dirk Reuyl and Eric Holmberg (famous for other things) and for 61 Cygni by K. A. Strand, who became in due course director of the US Naval Observatory. The mistake is an easy one to make. If you had before you the orbit of $\xi$ UMa that appears on page 682 of RDS, you might well be tempted to take a red pencil and connect up the dots from 1880 to 1899 and claim to have seen three cycles of a 5.9 year perturbation of the main 59.8 year period, with an amplitude that would imply a perturbing mass of about 0.05 times the mass of the secondary star.

The last in this lugubrious list is Peter (Piet) van de Kamp, who was director of Sproul Observatory (Swarthmore College) from 1937 to 1972, though he continued research there for some years, returning to Holland only very late in life. He sought through most of that time companions to nearby low-mass stars, and indeed greatly increased our knowledge of visual binaries, but now is almost entirely remembered for the claim of two planets orbiting Barnard's star. This was at one time a major topic of discussion, and I remember a boy of about 10 coming up to me after a very non-technical talk for a 3rd grade class somewhere around 1983 and asking, verbatim, "What is the current status of the companions of Barnard's star?" For van de Kamp to nearly the end of his life (van de Kamp 1986) they had masses of 0.7 and $0.5 M_{J}$ and orbit periods of 12 and 20 years. But a long data set from Leander McCormick Observatory found no trace of either, and the astronomical community came to blame a shift in the declination axis of the Sproul telescope and other mechanical and observational problems for the apparent wiggles in the sky.

The two "astrometry heroes" whose pictures I showed were Strand and van de Kamp, for obvious reasons. But, in addition, Strand had offered me a job at USNO in 1968 to work on the observatory's 2-micron survey (which proved not very informative). And at high school graduation in 1961, Ruth Staff Halliday, an unforgettable teacher of US history at Hollywood High School, had urged me to go to her alma mater, Swarthmore, which had offered a full scholarship. UCLA, where George Abell was the undergraduate advisor, was definitely a better choice for a potential astronomer.

\section{Conclusion}

If there is a moral to these folk tales, it has three parts:

- Observers be careful, the theorists are watching you;

- Theorists take care, the observers are watching you; and

- Both observers and theorists be warned the historians are watching you.

\section{Acknowledgement}

I am grateful to the organizers for the opportunity to put my thoughts on these subjects together for the IAU Symposium 314, to Ms. Alison Lara for her, as always, expert, 
transformation of a typed original into a keyboarded, formatted compuscript, and to all the colleagues living and dead from whom I have ruthlessly plagiarized this information. To the best of my knowledge, everything here is at least broadly true (as Stephen Hawking is supposed to have said about the film Theory of Everything) but it could be better documented and perhaps some day will be.

\section{References}

Bailes, M., Lyne, A. G., \& Shemar, S. L. 1991, Nature, 352, 311

Blaauw, A. 1952, BAN, 11, 405

Black, D. C. 1995, ARAEAA, 33, 359

, Borucki, W. J. 2015. Rep. Prog. Physics (in press)

Borucki, W. J. \& Summers, A. L. 1984, Icarus, 58, 121

Campbell, B., Walker, G. A. H., \& Yang, S. 1988, ApJ, 331, 902

Charbonneau, D., Brown, T. M., Dunham, E. W., et al. 2004, in The Search for Other Worlds, S. S. Holt \& D. Deming, eds., AIP Conf. Proc. 713, 151

Eggen, O. J. 1957, The Observatory, 77, 229

Eggen, O. J. 1965, in Galactic Structure, A. Blaauw \& M. Schmidt, eds., 111

Eggen, O. J. 1970, PASP, 82, 99

Eggen, O. J. 1993, ARAEAA, 31, 1

Eggen, O. J. 1998, AJ, 115, 2397

Jacobs, W. S. 1855, Plurality of Worlds, and MNRAS, 15, 228

Jones, H. R. A. 2004, in The Search for Other Worlds, S. S. Holt \& D. Deming, eds., AIP Conf. Proc. 713,17

Kapteyn, J. 1905. Rep. Br. Assoc., p. 257

Latham, D. W., Stefanik, R. P., Mazeh, T., Mayor, M., \& Burki, G. 1989, Nature, 339, 38

Marcy, G. W. \& Butler, R. P. 1996, ApJL, 464, L147

Mayor, M. \& Queloz, D. 1995, Nature, 378, 355

Navarro, J. F., Helmi, A., \& Freeman, K. C. 2004, ApJL, 601, L43

Oort, J. H. 1926, The Observatory, 49, 302

Oort, J. H. 1932, BAN, 6, 249

Proctor, R. 1869, Proc. Roy. Soc. 18, 169

Proctor, R. 1870, Phil. Trans. Roy. Soc.

Russell, H. N., Dugan, R. S., \& Stewart, R. M. 1927, JRASC, 21, 119

Schwarzschild, K. 1907, 1908. Goettingen Nachrichten

Schwarzschild, K. \& Hertzspring, E. 1939. Astron. Nach. 196, 9

See, T. J. J. 1896, AJ, 16, 17

See, T. J. J. 1899, AJ, 20, 33

Torres, G., et al. 2004, in The Search for Other Worlds, S. S. Holt \& D. Deming, eds., AIP Conf. Proc. 713,165

Trimble, V. 1997, in Star Formation Near and Far, S.S. Holt \& L.G. Mundy, eds., AIP Conf. Ser. 393,15

Trimble, V. 2004, in The Search for Other Worlds, S. S. Holt \& D. Deming, eds., AIP Conf. Proc. 713, 3

Trimble, V., et al. 2001, PASP, 113, 131

van de Kamp, P. 1986, Space Science Rev., 43, 211

Wolszczan, A. \& Frail, D. A. 1992, Nature, 355, 145

Zuckerman, B. \& Song, I. 2004, ARA\&A, 42, 685

Zuckerman, B. 2015, in 19th European Workshop on White Dwarfs, P. Dufour et al., eds., ASP Conf. Ser. 493, 291 